\title{
Nucleic acid detection and quantitative analysis of influenza virus using exhaled breath condensate
}

\author{
Xiaoguang $\mathrm{Li}^{1}$, Minfei Wang ${ }^{2}$, jing $\operatorname{chen}^{1}$, Fei $\operatorname{Lin}^{1}$, and Wei Wang ${ }^{1}$ \\ ${ }^{1}$ Peking University Third Hospital \\ ${ }^{2}$ Peking University
}

August 17, 2020

\begin{abstract}
Background: Influenza is a common respiratory infectious disease that can be transmitted by respiratory droplets from person to person. There were few researches on influenza virus in exhaled breath. This study aimed to investigate the detection of influenza virus in exhaled breath condensate (EBC) and conduct quantitative analysis to evaluate the existence and content of influenza virus in breath. Methods: We recruited 30 patients with respiratory tract infection in the 2019 influenza season. The clinical data and samples of nasal swabs were collected and rapid influenza diagnostic (antigen) tests (RIDTs) and EBC influenza virus nucleic acid detection were performed. Each sample was assessed thrice for qualitative and quantitative analysis. The patients were followed up after one month, and the EBC was assessed again. Results: The 30 cases were assessed for the presence of influenza virus nucleic acid in each EBC sample three times. The results showed that seven cases were positive for influenza A/ three times, seven cases were positive for influenza A twice, seven cases were positive for influenza A once, two cases were positive for influenza B once, and seven cases were negative for all three times. Furthermore, quantitative analysis showed that the influenza viral load in the EBC ranged from 103 to 107 copy/ml. Conclusion: The influenza virus can be detected using EBC and the concentration can be calculated. Thus, EBC can be used for molecular diagnosis of influenza.
\end{abstract}

\section{Nucleic acid detection and quantitative analysis of influenza virus using exhaled breath con- densate}

Running title: Quantitative analysis of influenza virus with EBC

Xiaoguang $\mathrm{Li}^{\mathrm{a} \#}$, Minfei Wang ${ }^{\mathrm{b} \#}$, Jing Chen ${ }^{\mathrm{a}}$, Fei Lin ${ }^{\mathrm{a}}$, Wei Wang ${ }^{\mathrm{a}}$

${ }^{a}$ Department of Infectious Diseases, Peking University Third Hospital, Beijing 100191, People's Republic of China

b State Key Joint Laboratory of Environmental Simulation and Pollution Control, College of Environmental Sciences and Engineering, Peking University, Beijing 100871, People's Republic of China

\# These authors contributed equally

Email addresses: Xiaoguang Li: lixiaoguangpuh3@bjmu.edu.cn, Minfei Wang: mingfeiwang@pku.edu.cn, Jing Chen: cj850101@qq.com, Fei Lin: leaflin66@163.com, Wei Wang: brown@bjmu.edu.cn

Correspondence should be addressed to Xiaoguang Li, Department of Infectious Diseases, Peking University Third Hospital, Beijing 100191, P.R. China, Tel: 86-10-13520004917, Email:lixiaoguangpuh3@bjmu.edu.cn

\section{Acknowledgments}


The authors thank Weiwei Li and Ju Wang from Shanghai Xingyao Medical Technology Development Co., Ltd, for their support in experiment technology. The authors thank Maosheng Yao from State Key Joint Laboratory of Environmental Simulation and Pollution Control, College of Environmental Sciences and Engineering, Peking University, for his support and suggestions about the study.

\section{Abstract :}

Background : Influenza is a common respiratory infectious disease that can be transmitted by respiratory droplets from person to person. There were few researches on influenza virus in exhaled breath. This study aimed to investigate the detection of influenza virus in exhaled breath condensate (EBC) and conduct quantitative analysis to evaluate the existence and content of influenza virus in breath.

Methods : We recruited 30 patients with respiratory tract infection in the 2019 influenza season. The clinical data and samples of nasal swabs were collected and rapid influenza diagnostic (antigen) tests (RIDTs) and EBC influenza virus nucleic acid detection were performed. Each sample was assessed thrice for qualitative and quantitative analysis. The patients were followed up after one month, and the EBC was assessed again.

Results : The 30 cases were assessed for the presence of influenza virus nucleic acid in each EBC sample three times. The results showed that seven cases were positive for influenza A/ three times, seven cases were positive for influenza A twice, seven cases were positive for influenza A once, two cases were positive for influenza B once, and seven cases were negative for all three times. Furthermore, quantitative analysis showed that the influenza viral load in the EBC ranged from $10^{3}$ to $10^{7}$ copy $/ \mathrm{ml}$.

Conclusion : The influenza virus can be detected using EBC and the concentration can be calculated. Thus, EBC can be used for molecular diagnosis of influenza.

Keywords : Exhaled breath condensate; Influenza; Detection; Molecular diagnosis

\section{Introduction}

Exhaled breath condensate (EBC) is a promising source of biomarkers of lung disease. However, EBC is not a biomarker, but a matrix in which biomarkers may be identified. ${ }^{1}$ Since the year 2000, EBC has been used to detect several lung diseases, such as asthma, chronic obstructive pulmonary disease, and lung cancer. Moreover, respiratory monitoring devices are constantly being updated ${ }^{2}$ and the metabolomics of EBC also shows the potential for early diagnosis. ${ }^{3}$

Influenza is a common respiratory infectious disease that can be transmitted by respiratory droplets from person to person. Lindsley reported that influenza viruses can be transmitted through aerosols when coughing and exhaling. ${ }^{4}$ Viable influenza A virus was detected more often in cough aerosol particles than in exhalation aerosol particles, but the difference was not large owing to individuals breathing more often than coughing. Xie reported that air sampling, as a surveillance tool for monitoring influenza activity in public locations, may provide early detection signals on influenza viruses that circulate in the community. ${ }^{5}$ Zhao reported that airborne transmission may have played a role in the spread of the 2015 highly pathogenic avian influenza outbreaks in the United States. ${ }^{6}$ In addition, during the influenza season, health facilities are crowded, increasing the risk of influenza transmission.

Before this study, our research team conducted several expiratory condensate studies. Equipment development using the Silicon Nanowire Sensor facility detected EBC H1N1 influenza virus. ${ }^{7}$ Experimental data revealed that bacteria and viruses in EBC can be rapidly collected using the method developed, with an observed efficiency of $100 \mu \mathrm{l} \mathrm{EBC}$ within 1 min. Culturing, DNA staining, SEM, and qPCR methods all detected high bacterial concentrations up to $7,000 \mathrm{CFU} / \mathrm{m}^{3}$ in $\mathrm{EBC}$, including both viable and dead cells of various types. Influenza A H3N2 virus was also detected in one EBC sample. ${ }^{8}$ We used a newly developed protocol of integrating an EBC collection device (PKU BioScreen) and loop-mediated isothermal amplification (LAMP) to investigate which bacterial pathogens are exhaled by humans, for example Haemophilus influenzae, Pseudomonas aeruginosa, Escherichia coli ,Staphylococcus aureus and Methicillin-resistantStaphylococcus aureus (MRSA). ${ }^{9}$ We also studied volatile organic compounds (VOCs) in EBC, and the differences in exhaled VOCs 
in patients with upper respiratory tract infection and healthy individuals. ${ }^{10}$ In this study, we focused on EBC influenza virus.

There is a need for a non-invasive and convenient testing method for respiratory viruses, such as the influenza virus. The EBC is non-invasive and easy to repeat as a new sampling option for children, the elderly, and people unable to tolerate other sampling methods. However, little research has been conducted on the ability of EBC to detect influenza viruses.

In this study, we investigated whether $\mathrm{EBC}$ can be used for the detection of influenza virus, using quantitative analysis. We believe this study could be a good reference for further studies on other respiratory infectious diseases, especially the novel severe acute respiratory syndrome coronavirus 2 (SARS-CoV-2).

\section{Research objectives and methods}

\subsection{Research objectives}

In the 2019 spring influenza season (from March 5 to April 8, 2019), 31 patients were chosen from the Department of Infectious Diseases, Peking University Third Hospital; however, one patient was omitted (lacking clinical data); thus 30 patients were finally enrolled for the study. The inclusion criteria were (1) fever $\mathrm{T}>37.2^{\circ} \mathrm{C}$; (2) cough and/or sore throat; and (3) age range between 18-65. After one month, all patients had a follow up assessment, and 17 patients agreed to the collection and re-testing of EBC.

All study participants provided informed consent, and the study design was approved by the Peking University Third Hospital Medical Science Research Ethics Committee 2017 (011-02).

\subsection{Research methods}

\subsubsection{Specimen collection}

Nasal swab collection : Keeping the head still, secretions were removed from the surface of the anterior nasal orifices. We gently inserted swab through nasal cavity to the nasopharynx; held for a few seconds, rotated gently, and removed the swab to transport media solution.

Collection of EBC : The patient was asked to exhale for 5 min. Approximately $500 \mu$ EBC was collected using the breath condensate collection device developed by the bioaerosol research group of Peking University. ${ }^{7}$

The collection steps are as follows: (1) The customised ice box is wiped with alcohol-soaked cotton to sterilise and conduct ultra-low temperature treatment $\left(-70^{\circ} \mathrm{C}\right)$. (2) A layer of sterile ultra-hydrophobic membrane, also treated with ultra-low temperature, was placed on the sterile ice box, the top cover of the sampling box was covered, and a sterile blowing pipe was inserted into the exhalation inlet. The subjects were asked to exhale steadily and slowly at a normal breathing rate for 5 min. (3) After collection, the super hydrophobic membrane was removed in a sterile environment. The exhaled condensate beads on the membrane surface were dragged back and forth with the sterile gun head and transferred to a sterile centrifuge tube to complete the collection process. The samples were stored at $-20{ }^{\circ} \mathrm{C}$ before testing.

\subsubsection{Reagent and method}

\section{Rapid detection of influenza virus antigen}

We used the Guangzhou Wanfu Biotechnology Co., Ltd. (Guangzhou city, Guangdong Province, China) influenza A/B antigen detection kit (colloidal gold method). An $80 \mu \mathrm{L}$ sample was added to the sample chamber in the test card to dissolve the transfer preservation solution of the sample to be tested; the results were observed within 15-20 min.

\section{Influenza virus nucleic acid detection Methods}

Each sample was assessed three times: twice using an influenza A/B virus RNA detection kit, Suzhou Tianlong Biotechnology Co., Ltd.(Suzhou city, Jiangsu Province, China), (called Tianlong), and once using 
influenza A/B virus nucleic acid detection kit (fluorescence PCR) Jiangsu Shuoshi Biotechnology Co., Ltd. (Taizhou city, Jiangsu Province, China), (called Shuoshi).

\section{Detection method:}

Tianlong : The RNA of influenza A/B virus was detected using a fluorescence PCR method; $8 \mu \mathrm{L}$ of $\mathrm{EBC}$ was directly added into $32 \mu \mathrm{L}$ reaction solution and was detected using theABI7500 fluorescence PCR instrument(Applied Biosystems, Seymour Fisher Technology (China) Co., Ltd, shanghai, China).Negative and positive results were determined by analysing the cycle threshold $(\mathrm{Ct})$ value. FAM and VIC were report fluorescent channels, which labelled with influenza A and B probes, respectively. The FAM channel, when $\mathrm{Ct}[?] 37.0$, means influenza A virus RNA was positive, whereas the VIC channel, when Ct[?]37.0, means influenza B virus RNA was positive.

Shuoshi : The RNA of influenza A/B virus was detected using a fluorescence PCR method; $5 \mu \mathrm{L}$ EBC was directly added into $20 \mu \mathrm{L}$ reaction solution and was detected using the ABI7500 fluorescence PCR instrument. The negative and positive results were determined by analysing the $\mathrm{Ct}$ value. The FAM channel, when Ct[?]34.7, means influenza A virus RNA was positive, whereas the VIC channel, when Ct[?]34.8, means influenza B virus RNA was positive.

\section{Determination of the quantitative value of the Tianlong kit:}

Influenza A/B plasmid was diluted to $1.0 \times 10^{9}$ as original concentration. Thereafter, it was diluted 10times to six gradients, which were named $\mathrm{E} 8^{\sim} \mathrm{E} 3$, and $\mathrm{F} 8^{\sim} \mathrm{E} 3$. These were taken as the standard point;Table 1 shows the specific concentrations. Fluorescence PCR was used to detect the linear relationship between the $\mathrm{Ct}$ value and the logarithm of plasmid copy number, and the sample concentration was determined through the experiment.

\subsection{Clinical data collection}

Demographic data such as gender and age of the patients, clinical data of the patients, blood routine, and influenza virus antigen test results were collected.

\subsection{Statistical analysis}

Statistical analysis was performed using SPSS 17 (SPSS Inc. Chicago). The measured data are expressed as $\pm \mathrm{s}$. The counting data are expressed in percentage.

\section{Results}

\subsection{Clinical data of 30 patients}

The patients' age ranged from 18 to 57 , with a median age of 23 . There were 15 males and 15 females (1:1 male-female ratio). The mean body temperature was $38.5 \pm 0.6^{\circ} \mathrm{C}\left(37.5-39.5^{\circ} \mathrm{C}\right)$. Results of blood routine examination were WBC $7.38 \pm 4.49 \times 10^{9} / \mathrm{L}$, normal in 26 cases, increased in two cases, decreased in one case, and absent in one case. The neutrophil percentage was $66.8 \% \pm 11.9 \%$, it increased in nine cases, decreased in two cases, and was absent in one case. The rapid influenza virus antigen detection test (colloidal gold method) showed four cases of influenza A, 12 cases of influenza B, and 14 cases were negative (See Table 2 ) .

\subsection{Detection results of EBC}

EBC nucleic acid detection was performed in 30 patients. Each sample was assessed three times, twice with Tianlong reagent and once with Shuoshi reagent. The results showed that seven cases were positive for influenza A three times, seven cases were positive for A twice, seven cases were positive for A once, two cases were positive for influenza B once, and seven cases were negative all three times.

If one sample had two or three times positive detection of nucleic acid, final nucleic acid test result was defined as positive, then the EBC nucleic acid test resulted in 14 cases $(14 / 30,46.7 \%)$ of influenza A. If 
one-time nucleic acid test is positive, the result is defined as positive. Further, the EBC nucleic acid test results had 21 cases of influenza $\mathrm{A}$ and two cases of influenza B.

If one nucleic acid test was positive, the nucleic acid was compared with the rapid detection of the influenza virus antigen. For influenza A, four cases were positive, and nine cases were negative by the two methods, with a consistent rate of $43.3 \%$ (13/30). For influenza B, two cases were positive, and 18 cases were negative by both methods, with a consistent rate of $66.7 \%$ (20/30) (See Table $\mathbf{2}$ ).

\subsection{Quantitative detection of influenza virus in EBC}

Since each sample was tested three times, there were three $\mathrm{Ct}$ values with similar results, we chose the $\mathrm{Ct}$ value of the first test only for further quantitative analysis to describe the range of concentrations at which influenza viruses can be detected in EBC. Based on the results of the first test with Tianlong reagent and the standard curve, the quantitative detection of the influenza virus showed that the influenza virus load in the EBC was between $10^{3}$ and $10^{7}$ copy $/ \mathrm{ml}$. (See Table 3 )

\subsection{Results of EBC in the 17 patients' follow up}

The results showed that 17 of the 30 patients with no symptoms were followed-up 1 month later and their EBC was collected. Table 4 shows that patients \#4, \#13, \#14, \#18, and \#27 results were negative for influenza $\mathrm{A}$ in the follow up. Patients \#12 and \#21 were still positive, \#17 went from negative to influenza A, and \#31 went from influenza B to influenza A; eight patients remained negative. (SeeTable 4 )

\section{Discussion}

Using EBC as the sample type for influenza virus detection and quantitative analysis is a novel method. In this study, we confirmed that influenza virus could be detected by EBC, and the viral load of influenza virus in EBC could be detected at $10^{3}-10^{7}$ copy $/ \mathrm{ml}$.

To date, there has been limited research using breath condensate testing for influenza viruses. George ${ }^{11}$ reported that in 19 influenza-like illnesses, influenza virus RNA was detected in nasopharyngeal swabs and EBC with 12 cases positive in nasopharyngeal swabs and one case positive in EBC. Moreover, Fabian ${ }^{12}$ reported four cases of influenza virus RNA in the EBC (three were influenza A virus and one was influenza B virus in 12 cases influenza-like illnesses). Houspie ${ }^{13}$ screened for 14 respiratory viruses in EBC samples from 102 upper respiratory infected people and detected six rhinoviruses and one influenza B virus. Costa $^{14}$ reported EBC detected respiratory herpes virus DNA with only 1 of the 24 samples being positive. However, the detection rates of the studies are not high owing to the low concentration of EBC virus. However, in this study, the patients had a relatively high viral load of $10^{3}-10^{7}$ copy $/ \mathrm{ml}$, which improved the detection rate of the influenza viruses in EBC, indicating EBC was a suitable influenza detection sample type.

The influenza detection methods such as rapid detection of influenza virus antigens have low sensitivity and affect the detection accuracy. Yarbrough ${ }^{15}$ reportedthat the sensitivity of rapid influenza diagnostic (antigen) tests (RIDTs) varies up to less than $50 \%$ and influenza B viruses are lower. This may be the reason for the low coincidence rate between the RIDT method and EBC nucleic acid detection in this study. The Food and Drug Administration (FDA) reclassified rapid influenza testing devices from Class I to Class II devices in 2018, requiring they must fulfil the lowest performance requirements and comply with additional controls. However, developing rapid molecular detection technology is needed and EBC will be one of the optional sample types.

Techniques for EBC collection and detection are improving.McDevitt ${ }^{16}$ reported development and performance evaluation of an exhaled breath bioaerosol collector for influenza and evaluated its protective measures. Furthermore, Fabian ${ }^{17}$ optimised the detection of influenza viruses and rhinoviruses in exhaled and airborne environments. These authors found that Trizol-chloroform extraction and MultiScribe RT increased virus detection by 10 -fold. 
In this study, we collected EBC in a safe and patient-friendly manner. We didnot require patients to open their mouths much to look directly at the pharynx for sampling, which can cause nausea or even spillage. Therefore, the EBC collection method has the advantages of being convenient, non-invasive,repeatable, and reducing the risk of exposure. Furthermore,the EBC method reduces the exposure of medical staff to patients during the pharyngeal and nasopharyngeal swab collection, and bronchoscope operations; in addition, it reduces the patient exposure to the environment by eliminating the need for open-mouth sampling in hospitals and medical institutions.

Furthermore, we tested the same sample three times, and the results showed a good consistency; this suggests other advantages of EBC.EBC have a relatively high viral load. The sample, which is in liquid form, is easy to handle, can be stored directly, homogenised, and directly used for detection. However, nasopharyngeal swabs need to be handled and diluted with preservation fluid, which is more complicated.

Pathogen collection of the EBC can cover the entire respiratory tract. However, the influenza virus typically occurs in the upper respiratory tract at the early stage and invades downwards with the progress of the illness. Severe influenza patients can have a negative throat swab of the upper respiratory tract but a positive alveolar lavage fluid. Thus, for patients with a negative throat swab who do not accept bronchoscopy and alveolar lavage fluid test, EBC provides a sample type selection increasing the chance of virus detection. We expect that the nucleic acid detection technology will continue to improve with detection becoming more sensitive, proving useful for influenza virus and novel coronavirus.

During the follow up assessments, two cases of EBC were still positive, indicating that asymptomatic infected people may exist with influenza virus, and the two cases of positive from negative may be new infections. Like the novel coronavirus, in the current study, asymptomatic infected people tested negative with multiple nucleic acid tests, but one month later, they tested positive after re-examination. Thus, further analysis and research are required toward asymptomatic carriers and their infection detection.

The limitations of the study, although EBC can be used for virus detection, there are no mature kits, methods, and gold standards, and the detection technical indicators still need investigation. In addition, there lacks matched comparisons with other sample types. Thus, it is necessary to expand the sample size and make further comparison of sample types to improve the detection technology. Moreover, the relationship between influenza viral load and prognosis can also be analysed according to the patients' disease course, illness condition, and repeated detection. The detection sample type used in this study showed that detection was possible for influenza and can provide a reference for studying the novel coronavirus.

\section{Data availability}

The data will be provided to others on reasonable requests to the corresponding author.

\section{Conflicts of interest}

The authors declare no conflict of interest.

\section{Funding statement}

The National Major Science and Technology Projects funded this project [project no. 2018ZX10732401-003], Beijing Haidian District Preventive Medicine Fund [grant no. 2017HDPMA04]

\section{Author contributions}

Xiaoguang $\mathrm{Li}$ is the principal clinical investigator, research designer, and thesis writer; Minfei Wang is the main experimenter and EBC collector; Jing Chen is an associate clinical investigator; Fei Lin performed clinical tests; Wei Wang performed clinical tests.

\section{References}

1. Davis MD, Montpetit AJ. Exhaled breath condensate.Immunol Allergy Clin North Am .2018;38(4):667678. doi:10.1016/j.iac.2018.06.002 
2. Maier D, Laubender E, Basavanna A, et al. Toward continuous monitoring of breath biochemistry: a paper-based wearable sensor for real-time hydrogen peroxide measurement in simulated breath.ACS Sens .2019;4(11):2945-2951.doi:10.1021/acssensors.9b01403

3. Beale D, Jones O, Karpe A, et al. A review of analytical techniques and their application in disease diagnosis in breathomics and salivaomics research. IJMS . 2016;18(1):24. doi:10.3390/ijms18010024

4. Lindsley WG, Blachere FM, Beezhold DH, et al. Viable influenza A virus in airborne particles expelled during coughs versus exhalations. Influenza Other Respi Viruses . 2016;10(5):404-413. doi:10.1111/irv.12390

5. Xie C, Lau EHY, Yoshida T, et al. Detection of influenza and other respiratory viruses in air sampled from a university campus: a longitudinal study. Clin Infect Dis . Published online April 9, 2019:ciz296. doi:10.1093/cid/ciz296

6. Zhao Y, Richardson B, TakleE, Chai L, Schmitt D, Xin H. Airborne transmission may have played a role in the spread of 2015 highly pathogenic avian influenza outbreaks in the United States.Sci Rep .2019;9(1):11755. doi:10.1038/s41598-019-47788-z

7. Shen F, Wang J, Xu Z, et al. Rapid flu diagnosis using silicon nanowire sensor. Nano Lett . 2012;12(7):37223730. doi:10.1021/nl301516z

8. Xu Z, Shen F, Li X, et al. Molecular and microscopic analysis of bacteria and viruses in exhaled breath collected using a simple impaction and condensing method. Poon LLM, ed.PLoS ONE . 2012;7(7):e41137. doi:10.1371/journal.pone.0041137

9. Zheng Y, Chen H, Yao M, Li X. Bacterial pathogens were detected from human exhaled breath using a novel protocol. J Aerosol Sci .2018;117:224-234.doi:10.1016/j.jaerosci.2017.12.009

10. Wang J, Zheng Y, Lui Z, Yao M, Li X, et al. Differences in Exhaled VOCs from Patients with Upper Respiratory Tract Infection and Healthy Ones. Acta Scientiarum Naturalium Universitatis Pekinensis . 2018;54(4):807-813.doi:10.13209/j.0479-8023.2017.191

11. St. George K, Fuschino ME, Mokhiber K, Triner W, Spivack SD. Exhaled breath condensate appears to be an unsuitable specimen type for the detection of influenza viruses with nucleic acid-based methods. $J$ Virol Methods . 2010;163(1):144-146. doi:10.1016/j.jviromet.2009.08.019

12. Fabian P, McDevitt JJ, DeHaan WH, et al. Influenza virus in human exhaled breath: an observational study. Fouchier RAM, ed.PLoS ONE . 2008;3(7):e2691. doi:10.1371/journal.pone.0002691

13. Houspie L, De Coster S, Keyaerts E, et al. Exhaled breath condensate sampling is not a new method for detection of respiratory viruses. Virol $J$.2011;8(1):98.doi:10.1186/1743-422X-8-98

14. Costa C, Bucca C, Bergallo M, Solidoro P, Rolla G, Cavallo R. Unsuitability of exhaled breath condensate for the detection of Herpesviruses DNA in the respiratory tract. J Virol Methods . 2011;173(2):384-386. doi:10.1016/j.jviromet.2011.02.004

15. Yarbrough ML, Burnham C-AD, Anderson NW, et al. Influence of molecular testing on influenza diagnosis. Clin Chem . 2018;64(11):1560-1566. doi:10.1373/clinchem.2018.289363

16. McDevitt JJ, Koutrakis P, Ferguson ST, et al. Development and performance evaluation of an exhaled breath bioaerosol collector for influenza virus. Aerosol Sci Technol . 2013;47(4):444-451. doi:10.1080/02786826.2012.762973

17. Fabian P, McDevitt JJ, Lee W-M, Houseman EA, Milton DK. An optimized method to detect influenza virus and human rhinovirus from exhaled breath and the airborne environment. $J$ Environ Monit . 2009;11(2):314-317. doi:10.1039/B813520G

\section{Hosted file}


table 1.docx available at https://authorea.com/users/351375/articles/476018-nucleic-aciddetection-and-quantitative-analysis-of-influenza-virus-using-exhaled-breath-condensate

\section{Hosted file}

table 2.docx available at https://authorea.com/users/351375/articles/476018-nucleic-aciddetection-and-quantitative-analysis-of-influenza-virus-using-exhaled-breath-condensate

\section{Hosted file}

table 3.docx available at https://authorea.com/users/351375/articles/476018-nucleic-aciddetection-and-quantitative-analysis-of-influenza-virus-using-exhaled-breath-condensate

\section{Hosted file}

table 4.docx available at https://authorea.com/users/351375/articles/476018-nucleic-aciddetection-and-quantitative-analysis-of-influenza-virus-using-exhaled-breath-condensate 